\title{
Multiple scattering of polarized radiation by non-spherical grains: First results
}

\author{
S. Wolf ${ }^{1}$, N. V. Voshchinnikov ${ }^{2}$, and Th. Henning ${ }^{3}$ \\ 1 Thuringian State Observatory Tautenburg, Sternwarte 5, 07778 Tautenburg, Germany \\ 2 Sobolev Astronomical Institute, St. Petersburg University, Universitetskii Prosp. 28, \\ 198504 Stary Peterhof-St. Petersburg, Russia and Isaac Newton Institute of Chile, St. Petersburg Branch, \\ Russia \\ 3 Astrophysical Institute and University Observatory (AIU), Friedrich Schiller University, Schillergäßchen 3, \\ 07745 Jena, Germany
}

Received 29 December 2000 / Accepted 21 January 2002

\begin{abstract}
We present the first numerical radiative transfer simulation of multiple light scattering in dust configurations containing aligned non-spherical (spheroidal) dust grains. Such models are especially important if one wants to explain the circular polarization of light, observed in a variety of astronomical objects. The optical properties of the spheroidal grains are calculated using the method of separation of variables developed by Voshchinnikov \& Farafonov (1993). The radiative transfer problem is solved on the basis of the Monte Carlo method. Test simulations, confirming the correct numerical implementation of the scattering mechanism, are presented. As a first application, we investigate the linear and circular polarization of light coming from a spherical circumstellar shell. This shell contains perfectly aligned prolate or oblate spheroidal grains. We investigate the dependence of the results on the grain parameters (equivolume radius, aspect ratio) and the shell parameters (inner/outer radius, optical thickness). The most remarkable features of the simulated linear polarization maps are so-called polarization null points where the reversal of polarization occurs. They appear in the case when the grain alignment axis is perpendicular to the line of sight. The position of these points may be used for the estimation of grain shape and geometrical structure of the shell. The origin of null points lies in the physics of light scattering by non-spherical particles and is not related to the cancellation of polarization as was discussed in previous models. The maps of circular polarization have a sector-like structure with maxima at the ends of lines inclined to the grain alignment axis by $\pm 45^{\circ}$.
\end{abstract}

Key words. polarization - radiative transfer - scattering - methods: numerical - (stars:) circumstellar matter ISM: clouds

\section{Introduction}

It is now well established that the polarization of optical and near-infrared radiation from young and evolved stellar objects, reflection nebulae, and active galactic nuclei is mainly caused by dust grains. In many cases the observed polarization can be satisfactorily interpreted by light scattering on spherical grains. In particular, the orientation of the polarization vectors is often used for the investigation of the dust distribution and for identifying the location of the embedded illuminating source(s).

However, even in the case of simple objects like ordinary reflection nebulae, deviations of polarization vectors from the direction perpendicular to a star were discovered more than three decades ago (Elvius \& Hall 1967). These deviations are displayed in the outer filamentary

Send offprint requests to: N. V. Voshchinnikov, e-mail: nvv@astro.spbu.ru parts of the Merope nebula and may be easily explained by light scattering on non-spherical grains aligned by a magnetic field along the filaments. Non-centrosymmetric polarization patterns have been observed in bipolar and cometary nebulae (Scarrott et al. 1989), young stellar objects (Hajjar \& Bastien 1996), evolved stars (Kastner \& Weintraub 1996), and are also clearly seen in polarization maps of comets (Dollfus \& Suchail 1987). They may be attributed to non-spherical grains, although they could be caused by multiple scattering on spherical particles as well. Another effect which may be related to the light scattering by non-spherical grains is the wavelength dependence of the positional angle of polarization observed in red giants, AGB stars, and bipolar reflection nebulae (see, e.g., Johnson \& Jones 1991). The variations from blue to red may reach $20^{\circ}-60^{\circ}$ and it is very difficult or even impossible to interpret this behaviour using spherical grains only. 
Recently, very high degrees of circular polarization of scattered light in the Orion molecular cloud were measured by Chrysostomou et al. (2000). The authors suggest that the circular polarization is produced by aligned non-spherical grains. Multiple scattering of radiation by spherical or randomly oriented non-spherical grains results in a much smaller circular polarization degree than observed. In addition, the interstellar polarization and polarized thermal emission phenomena prove that nonspherical grains exist in the interstellar medium. These effects arise because of dichroic extinction/emission of radiation by aligned non-spherical grains and were modeled with spheroidal grains (Voshchinnikov 1990; Kim \& Martin 1995; Onaka 2000).

Up to now, spheroidal grains have been used for the interpretation of scattered radiation only in the case of single scattering (Voshchinnikov 1998; Gledhill \& McCall 2000). The numerical simulation of the polarized radiation transfer through a medium with non-spherical grains, including multiple scattering, is extremely difficult. In the case of spherical grains nearly all previous simulations are based on Monte Carlo simulations. This method was used for the interpretation of polarimetric observations of various stars (see, e.g., Daniel 1980; Voshchinnikov \& Karjukin 1994) as well as the production of polarimetric maps of different extended objects (e.g., Bastien \& Ménard 1988; Fischer et al. 1994; Wolf et al. 1998). The most recent, major achievement of the Monte Carlo technique - in respect to the solution to the radiative transfer (RT) problem - is the development of RT codes that allow to calculate the spectral energy distribution of multi-dimensional dust configurations self-consistently (Wolf et al. 1999; Wolf \& Henning 2000).

The main aim of this paper is to provide the first radiative transfer simulations including scattering, extinction, absorption, and re-emission of radiation by aligned spheroidal dust grains. Our formalism will be applied to several simple model configurations. Because of the complexity of the problem, we decided to consider the separate mechanisms step by step. In this paper, the basic theory is presented and the main numerical features of the simulation are described (Sect. 2). Furthermore, we consider multiple scattering of light by spheroidal grains and the resulting linear and circular polarization (Sects. 3 and 4).

\section{Basics}

\subsection{Dust grain parameters}

Let us suppose that the dust grains are prolate or oblate homogeneous spheroids with an aspect ratio $a / b$, where the quantities $a$ and $b$ are the major and minor semiaxes of a spheroid, respectively. The particle size is characterized by the radius $r_{\mathrm{V}}$ of a sphere with the same volume as that of the spheroid (equivolume radius). The major semiaxis $a$ of the spheroid is connected with $r_{\mathrm{V}}$ by

$a=r_{\mathrm{V}} \cdot\left(\frac{a}{b}\right)^{2 / 3}$ for prolate spheroids and

$a=r_{\mathrm{V}} \cdot\left(\frac{a}{b}\right)^{1 / 3}$

for oblate spheroids. The optical properties of a particle also depend on the complex refractive index of the grain material $m_{\lambda}$ and the angle $\alpha$ between the rotation axis of the spheroid and the wave-vector direction (direction of the incident radiation; $0^{\circ} \leq \alpha \leq 90^{\circ}$, see Fig. 1 ).

\subsection{Radiative transfer concept}

In this section we outline the main ideas of our solution of the RT problem taking into account multiple scattering and dichroic absorption, and reemission by spheroidal grains. Our solution is based on the Monte Carlo method which is described only briefly here. For a more general overview about RT based on the Monte Carlo method, we refer to the papers of Wolf et al. (1999) and Wolf \& Henning (2000) (see also Fischer et al. 1994).

In our solution to the RT problem, the radiation energy is partitioned into so-called weighted photons (in the following we will use the term "photon"). The energy, intensity, and polarization of a weighted photon are described by its wavelength $\lambda$ and the Stokes vector $\hat{I}=(I, Q, U, V)^{\mathrm{T}}$. The transformation of the Stokes vector by the $i$ th scattering can be described by a $4 \times 4$ (Müller) matrix $\hat{F}$ :

$$
\left(\begin{array}{c}
I \\
Q \\
U \\
V
\end{array}\right)_{i} \propto \hat{F}\left(\begin{array}{c}
I \\
Q \\
U \\
V
\end{array}\right)_{i-1}
$$

The main features distinguishing the light scattering by aligned non-spherical particles and spherical particles are:

1. Azimuthal dependence of the scattered radiation;

2. Linear polarization in the forward and backward directions;

3. Deviation of the positional angle of linear polarization after first scattering from the direction perpendicular to the illuminating source and

4. Circular polarization after first scattering.

These features result from the fact that in the general case of non-spherical particles - in contrast to spherical grains all 16 elements of the scattering matrix are non-zero (see Voshchinnikov \& Farafonov 1993).

The spatial distribution of the illuminating star(s) and the dust grains is defined inside a convex model space. The free path length $l$ of a photon (distance between the point of emission and the first point of scattering/absorption or two points of scattering/absorption) is determined by the dust density distribution $n_{\mathrm{d}}\left(\boldsymbol{R}_{i}\right)$ at the coordinate $\boldsymbol{R}_{i}$ and the extinction cross-section for the transmitted radiation $\tilde{C}_{\text {ext }}\left(\boldsymbol{R}_{i}\right)$

$\tilde{\tau}_{\mathrm{ext}}=\sum_{i=1}^{i_{\mathrm{end}}} n_{\mathrm{d}}\left(\boldsymbol{R}_{i}\right) \cdot \tilde{C}_{\mathrm{ext}}\left(\boldsymbol{R}_{i}\right) \cdot \Delta l_{i}$. 
The quantity $i_{\text {end }}$ represents the number of integration steps along the free path length, and the quantity $\Delta l_{i}$ is the corresponding geometrical step width. The free mean path length

$l=\sum_{i=1}^{i_{\text {end }}} \Delta l_{i}$

can be derived from the optical depth $\tilde{\tau}_{\text {ext }}$ as follows

$\tilde{\tau}_{\text {ext }}=-\ln (1-\zeta)$.

Here, $\zeta$ is a random number uniformly distributed on the interval $[0,1]$ (see Wolf et al. 1999 for details).

The extinction cross-section $\tilde{C}_{\text {ext }}$ is calculated taking into account the state of polarization of the incident radiation (see, e.g., Martin 1974):

$$
\begin{aligned}
\tilde{C}_{\text {ext }}=\frac{1}{2}\left(C_{\text {ext }}^{\mathrm{TM}}+C_{\text {ext }}^{\mathrm{TE}}\right)+\frac{1}{2}\left(C_{\text {ext }}^{\mathrm{TM}}-C_{\text {ext }}^{\mathrm{TE}}\right) \cos 2 \Psi \frac{Q_{0}}{I_{0}} \\
+\frac{1}{2}\left(C_{\mathrm{ext}}^{\mathrm{TM}}-C_{\mathrm{ext}}^{\mathrm{TE}}\right) \sin 2 \Psi \frac{U_{0}}{I_{0}} .
\end{aligned}
$$

Here, $\Psi$ is the angle between the particle frame and the laboratory frame. $\left(I_{0}, Q_{0}, U_{0}, V_{0}\right)$ is the Stokes vector of the incident light. The extinction cross-sections $C_{\text {ext }}^{\mathrm{TE}, \mathrm{TM}}$ are connected with the efficiency factors $Q_{\mathrm{ext}}^{\mathrm{TE}, \mathrm{TM}}$ by

$C_{\mathrm{ext}}^{\mathrm{TE}, \mathrm{TM}}=G \cdot Q_{\mathrm{ext}}^{\mathrm{TE}, \mathrm{TM}}$,

where the quantity $G$ is the geometrical cross-section of the dust grain (the area of the particle shadow). The superscripts TM and TE are related to the two cases of the polarization of the incident radiation (TM and TE modes). The geometrical cross-section $G$ can be derived as follows

$G(\alpha)=\pi r_{\mathrm{V}}^{2} \cdot\left(\frac{a}{b}\right)^{-2 / 3} \cdot\left[\left(\frac{a}{b}\right)^{2} \sin ^{2} \alpha+\cos ^{2} \alpha\right]^{1 / 2}$

for a prolate spheroid and

$G(\alpha)=\pi r_{\mathrm{V}}^{2} \cdot\left(\frac{a}{b}\right)^{2 / 3} \cdot\left[\left(\frac{a}{b}\right)^{-2} \sin ^{2} \alpha+\cos ^{2} \alpha\right]^{1 / 2}$.

for an oblate spheroid. The efficiency factors $Q_{\text {ext }}^{\mathrm{TM}, \mathrm{TE}}$ are calculated from the solution to the light scattering problem for spheroids obtained by the method of separation of variables (see Voshchinnikov \& Farafonov 1993 for details).

We would like to note that the extinction cross-section $\tilde{C}_{\text {ext }}$ (see Eq. (7)) has to be determined at each integration step along the photon path (see Eq. (4)). In the case of the RT in a medium with aligned non-spherical grains, the step width along the photon free path is determined not only by the scale of the density structure (as for spherical particles) but also by the degree of spatial variations of the grain alignment. Owing to this and the more complex treatment of the scattering process compared with spherical grains, the total computation run-time is by an order of magnitude larger for aligned spheroids than for spheres.

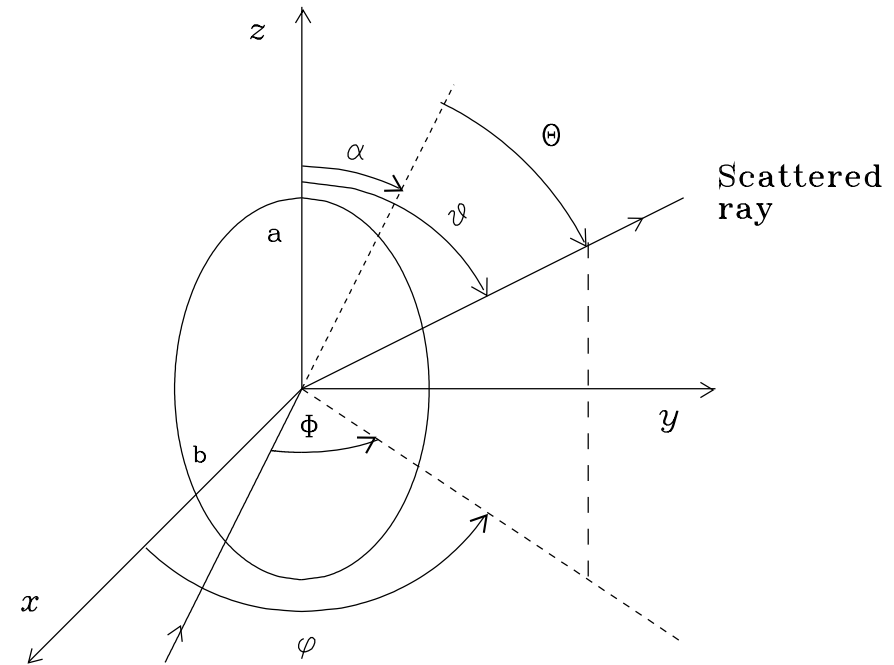

Incident ray

Fig. 1. Scattering geometry for prolate homogeneous spheroid. The origin of the coordinate system is at the centre of the spheroid while the $z$-axis coincides with its axis of revolution. The angle of incidence $\alpha$ is the angle in the $x-z$-plane between the direction of incidence and the $z$-axis. The scattered field in the far-field zone is represented in a spherical coordinate system by the angles $\theta, \varphi$ in the reference system related to the spheroid's rotation axis (particle frame) or by the angles $\Theta, \Phi$ in the reference system related to the scattering plane (laboratory frame).

Finally, depending on the model geometry and the dust density distribution, the photon leaves the model space after a certain number of interactions with dust grains (scattering, absorption). Then, it will be "observed" by array-detector-like planes arranged around the model space. The Stokes vector has to be projected onto the respective plane. For a detailed description of the projection formalism we refer to Fischer et al. (1994).

\subsection{Scattering}

The solution to the problem of light scattering by spheroidal particles is given in a particle reference system related to the rotation axis of a spheroid (in spherical coordinates $\theta, \varphi$ ), while the RT is considered in the laboratory reference system (in spherical coordinates $\Theta, \Phi$; see Fig. 1). The probability of a photon of being scattered into the direction $(\Theta, \Phi)$ is proportional to the value of the first element of the scattering matrix $F_{11}(\alpha, \Theta, \Phi)$. We transformed the scattering matrix from the particle to the laboratory frame. Then, the scattering matrix is calculated using the standard expressions (Bohren \& Huffman 1983). In order to check the transition from the particle system to the laboratory one, the transformation was also done for light scattering by spheroidal particles in the quasistatic approximation where the solution can be written in both coordinate systems (see Farafonov et al. 2001 for details). 


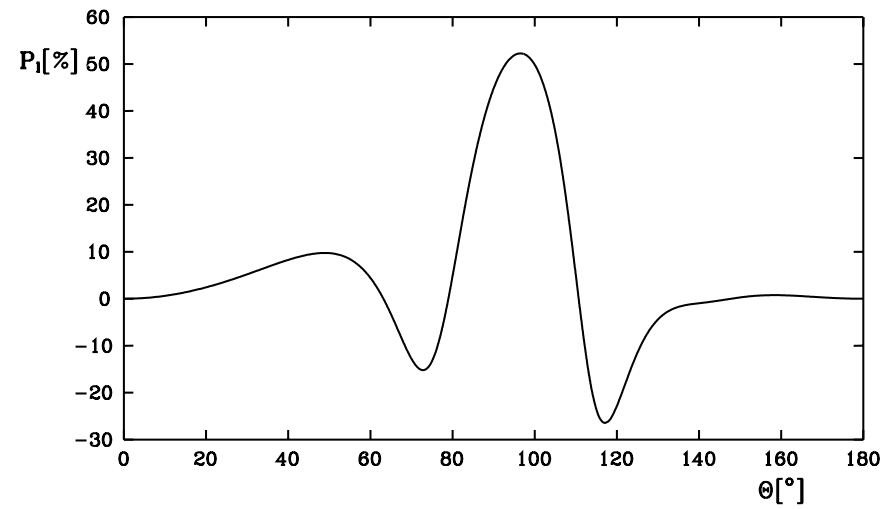

Fig. 2. The linear polarization of radiation $P_{1}(\Theta)$ after the first scattering by a prolate spheroidal particle with refractive index $m=1.7+0.03 i$ (typical of astronomical silicate at optical wavelengths), equivolume radius $r_{\mathrm{V}}=0.20 \mu \mathrm{m}$, aspect ratio $a / b=4$ and the incidence angle $\alpha=0^{\circ}$. The wavelength of incident radiation is $\lambda=0.628 \mu \mathrm{m}$. To avoid skipping of local minima/maxima a step size smaller than $5^{\circ}$ for $\Theta$ is required.

The elements of the scattering matrix were normalized using the standard procedure (Bohren \& Huffman 1983)

$\frac{2}{k^{2}\left(C_{\mathrm{sca}}^{\mathrm{TM}}+C_{\mathrm{sca}}^{\mathrm{TE}}\right)} \int_{0}^{2 \pi} \int_{0}^{\pi} F_{11}(\alpha, \Theta, \Phi) \sin \Theta \mathrm{d} \Theta \mathrm{d} \Phi=1$,

where $C_{\text {sca }}$ is the scattering cross-section and $k=2 \pi / \lambda$ the wavenumber. The correctness of these computations was verified using the relationships for matrices, describing scattering by small particles (see Hovenier \& van der Mee 2000 and references therein).

The calculation of the elements of the scattering matrix $\hat{F}\left(m, r_{\mathrm{V}}, a / b, \alpha, \Theta, \Phi\right)$ and efficiency factors $Q_{\mathrm{sca}, \mathrm{ext}}^{\mathrm{TM}, \mathrm{TE}}\left(m, r_{\mathrm{V}}, a / b, \alpha\right)$ for a single particle with the fixed equivolume radius $r_{\mathrm{V}}$, the aspect ratio $a / b$, and the refractive index $m$ requires several minutes of computer time ${ }^{1}$. These quantities were precalculated before starting the RT modelling. In order to guarantee that the main features (local minima/maxima) of the scattering matrix elements (and/or derived quantities, for instance, the polarization of singly scattered light) are present in the discrete distributions as a function of $\alpha, \Theta$, and $\Phi$ (see Figs. 2 and 3), we use step widths of $\Delta \alpha=\Delta \Theta=\Delta \Phi=2^{\circ}$ in our simulations (see Fig. 3).

\subsection{Absorption and reemission}

The absorption is described by the albedo $\Lambda$ of the particle which is defined as the ratio of the scattering and extinction cross sections. It is equal to

$\Lambda^{\mathrm{TM}, \mathrm{TE}}\left(m, r_{\mathrm{V}}, a / b, \alpha\right)=\frac{C_{\mathrm{sca}}^{\mathrm{TM}, \mathrm{TE}}\left(m, r_{\mathrm{V}}, a / b, \alpha\right)}{C_{\mathrm{ext}}^{\mathrm{TM}, \mathrm{TE}}\left(m, r_{\mathrm{V}}, a / b, \alpha\right)}$

\footnotetext{
1 A Pentium II $450 \mathrm{MHz}$ processor was used for our calculations.
}

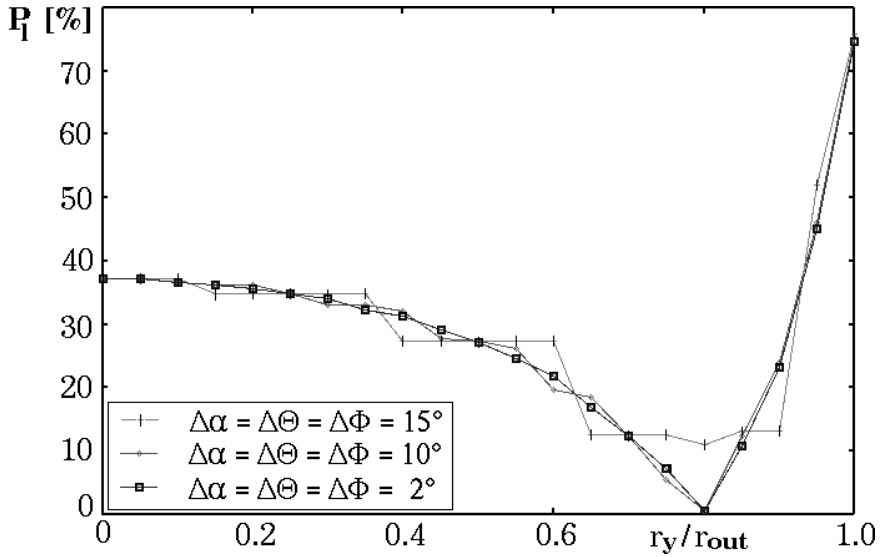

Fig. 3. The degree of linear polarization in the midplane of the polarization map shown in Fig. 5a in dependence of the projected distance. Three different discretizations for the angles $\alpha, \Theta$, and $\Phi$ were applied $\left(\Delta \alpha=\Delta \Theta=\Delta \Phi=2^{\circ}, 10^{\circ}\right.$, and $\left.15^{\circ}\right)$. Only the smallest step width gives a sufficient approximation to the analytical solution presented in Fig. 5b1 (see Sect. 2.3 for discussion).

for totally polarized light and

$$
\Lambda^{(0)}\left(m, r_{\mathrm{V}}, a / b, \alpha\right)=\frac{C_{\mathrm{sca}}^{\mathrm{TM}}+C_{\mathrm{sc}}^{\mathrm{TE}}}{C_{\mathrm{ext}}^{\mathrm{TM}}+C_{\mathrm{ext}}^{\mathrm{TE}}}
$$

for non-polarized light.

While in the case of absorption by spherical particles, the Stokes vector $\hat{I}$ can be multiplied by a scalar value of the albedo, for spheroidal particles the following $4 \times 4$ matrix is needed (in the particle frame):

$\hat{\Lambda}_{\text {part. }}=\left(\begin{array}{cccc}\tilde{l}_{1} & \tilde{l}_{2} & 0 & 0 \\ \tilde{l}_{2} & \tilde{l}_{1} & 0 & 0 \\ 0 & 0 & \tilde{l}_{1} & 0 \\ 0 & 0 & 0 & \tilde{l}_{1}\end{array}\right)_{\text {part. }}$

Here, the quantities $\tilde{l}_{1}$ and $\tilde{l}_{2}$ are defined as

$$
\begin{aligned}
\tilde{l}_{1}=\Lambda & =\frac{\Lambda^{\mathrm{TM}}}{1+C_{\mathrm{ext}}^{\mathrm{TM}} / C_{\mathrm{ext}}^{\mathrm{TE}}}+\frac{\Lambda^{\mathrm{TE}}}{1+C_{\mathrm{ext}}^{\mathrm{TE}} / C_{\mathrm{ext}}^{\mathrm{TM}}}, \\
\tilde{l}_{2} & =\frac{\Lambda^{\mathrm{TM}}}{1+C_{\mathrm{ext}}^{\mathrm{TM}} / C_{\mathrm{ext}}^{\mathrm{TE}}}-\frac{\Lambda^{\mathrm{TE}}}{1+C_{\mathrm{ext}}^{\mathrm{TE}} / C_{\mathrm{ext}}^{\mathrm{TM}}} .
\end{aligned}
$$

Before applying the albedo matrix $\hat{\Lambda}_{\text {part. }}$, it has to be rotated into the particle frame using the rotation matrix $\hat{R}$

$\hat{R}=\left(\begin{array}{cccc}1 & 0 & 0 & 0 \\ 0 & \cos 2 \Psi & \sin 2 \Psi & 0 \\ 0 & -\sin 2 \Psi & \cos 2 \Psi & 0 \\ 0 & 0 & 0 & 1\end{array}\right)$.

The absorption process can then be described by the expression

$\hat{I}_{\text {after }}=\hat{R}^{-1} \hat{\Lambda}_{\text {part. }} \hat{R} \hat{I}_{\text {before }}$

whereby $\hat{I}_{\text {before }}\left(\hat{I}_{\text {after }}\right)$ is the Stokes vector of the radiation before (after) absorption. 
In the case of (re)emission of radiation by a spheroidal particle, the Stokes vector of the photon can be written as follows (particle frame):

$$
\left(\begin{array}{c}
I_{0} \\
Q_{0} \\
U_{0} \\
V_{0}
\end{array}\right)_{\text {part. }} \propto\left(\begin{array}{c}
\left(C_{\mathrm{abs}}^{\mathrm{TM}}+C_{\mathrm{abs}}^{\mathrm{TE}}\right) / 2 \\
-\left(C_{\mathrm{abs}}^{\mathrm{TM}}-C_{\mathrm{abs}}^{\mathrm{TE}}\right) / 2 \\
0 \\
0
\end{array}\right)_{\text {part. }} B_{\lambda}\left(T_{\mathrm{d}}\right) .
$$

Here, $C_{\text {abs }}$ is the absorption cross-section, $B_{\lambda}\left(T_{\mathrm{d}}\right)$ the Planck function and $T_{\mathrm{d}}$ the grain temperature.

\section{Test simulations}

The best way to test any RT code is the comparison of its results with those obtained with another RT code/technique or an analytical solution. Because the RT code presented here is the first that provides the treatment of multiple scattering of polarized light by aligned non-spherical particles, a lack of suitable test cases for comparison is evident. Nevertheless, in the following we present test calculations which confirm the correct implementation of the new scattering mechanism into our RT code.

For test simulations we used particles with the following parameters:

- shape of the grains: oblate;

- refractive index: $m=1.7+0.03 i$;

- equivolume radius: $r_{\mathrm{V}}=0.1 \mu \mathrm{m}$;

- aspect ratio: $a / b=2.0$;

- wavelength of incident radiation: $\lambda=0.628 \mu \mathrm{m}$.

Then the grain size parameter is $x_{\mathrm{V}}=2 \pi r_{\mathrm{V}} / \lambda=1$.

\subsection{Single scattering in a geometrically and optically thin hemisphere}

Firstly, we consider single scattering in a thin layer of oblate spheroids at the surface of an hemisphere (see Fig. 4). The ratio of the inner radius $\left(r_{\text {in }}\right)$ of the shell to the outer one $\left(r_{\text {out }}\right)$ amounts to $r_{\text {in }} / r_{\text {out }}=0.99$. The non-rotating particles show a static picket fence orientation with their rotation axes being aligned parallel to the $z$-axis of the global coordinate system of the model space. The density profile $n(r)$ in the shell follows a power law:

$n(r) \propto r^{-1}$.

The optical thickness of the shell at the wavelength of the simulated RT $(\lambda=0.628 \mu \mathrm{m})$ is 0.2 along the $z$-axis, i.e. $\tau_{z}(0.628 \mu \mathrm{m})=0.2$. Because of the chosen grain alignment, $\tau_{z}$ is proportional to the extinction cross-section in the case of light incident parallel to the grain rotation axis, $\tau_{0}=\tau_{z} \propto C_{\text {ext }}\left(0^{\circ}\right)$. In other directions, the optical thickness of the shell may be found as $\tau_{\alpha}=$ $\tau_{z} C_{\text {ext }}(\alpha) / C_{\text {ext }}\left(0^{\circ}\right)$ and, in particular, along the line of sight $\left(x\right.$-axis) and the $y$-axis we have $\tau_{\mathrm{x}}=\tau_{y}=\tau_{90^{\circ}}=$ $\tau_{z} C_{\text {ext }}\left(90^{\circ}\right) / C_{\text {ext }}\left(0^{\circ}\right)$. Using Eq. (7) for non-polarized stellar radiation and the standard model described above, we
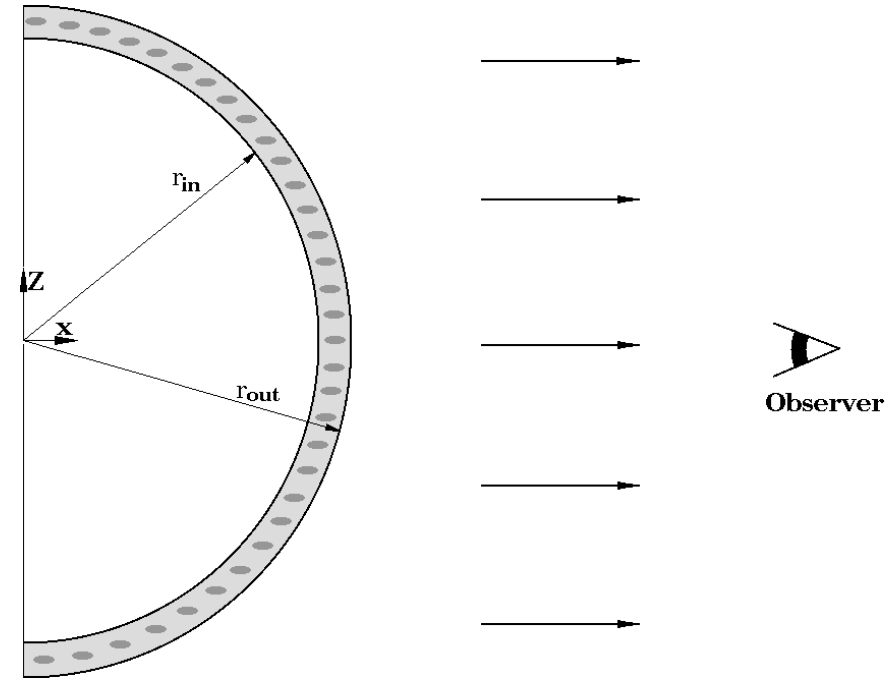

Fig. 4. Model configuration for comparison with the results of Matsumura \& Seki (1996). It consists of a thin dust layer at the surface of a hemisphere. The ratio of the inner radius to the outer one amounts to $r_{\text {in }} / r_{\text {out }}=0.99$. The shell contains oblate grains, whereby their rotation axes $(b)$ are oriented parallel to the $z$-axis. The dark grey oval pattern symbolizes the aligned grains. The configuration is shown for $y=0$ ( $x z$-plane, the azimuthal angle $\Phi=180^{\circ}$ ). An observer sees the radiation at the scattering angles $\Theta=0^{\circ}-90^{\circ}$. In this case the condition $\Theta+\alpha=90^{\circ}$ is satisfied in all points of the dust layer.

have $\tau_{90^{\circ}} \approx 0.35$ (prolate grains) and $\tau_{90^{\circ}} \approx 0.13$ (oblate grains), if $\tau_{0}(0.628 \mu \mathrm{m})=0.2$. For more elongated or flattened particles $(a / b=10)$, these values become equal to $\tau_{90^{\circ}} \approx 0.68$ and 0.082 for prolate and oblate grains, respectively.

In contrast to polarization maps obtained for model configurations with the same geometry, but containing spherical dust grains, the polarization pattern is not centro-symmetric (see Fig. 5a). This is because of the azimuthal asymmetry of light scattering by aligned nonspherical particles. The net polarization in this case is not equal to zero. We compare our results with those obtained by Matsumura \& Seki $(1996)^{2}$. In Fig. 5 the comparison of the polarization degree as a function of the projected distance from the centre along three different lines in the polarization map is shown. These graphs as well as the polarization pattern (see for comparison Fig. 2 from Matsumura \& Seki 1996) are in perfect agreement.

Qualitatively, the polarization pattern is quite similar to that of a young stellar object surrounded by a spherical shell and an optically thick disk seen edge-on. There the parallel polarization pattern is due to light scattering on the disk surface while the centro-symmetric polarization pattern is due to light scattering in the circumstellar environment. Corresponding RT simulations with spherical dust grains have been performed by Ménard (1988) and Fischer et al. (1994). The most interesting feature of the pattern is the presence of polarization null points (see

\footnotetext{
2 The numerical data in tabular form were provided by Matsumura (1999).
} 
A

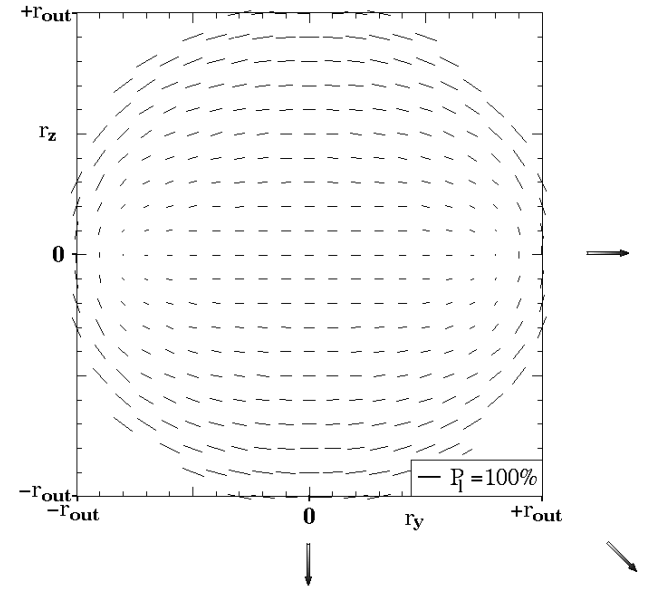

B2

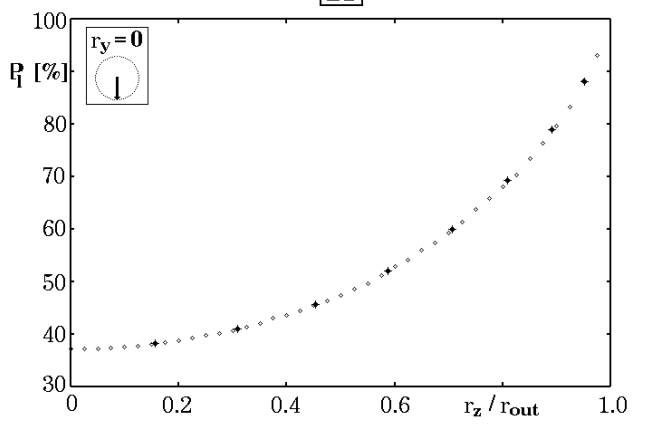

B1
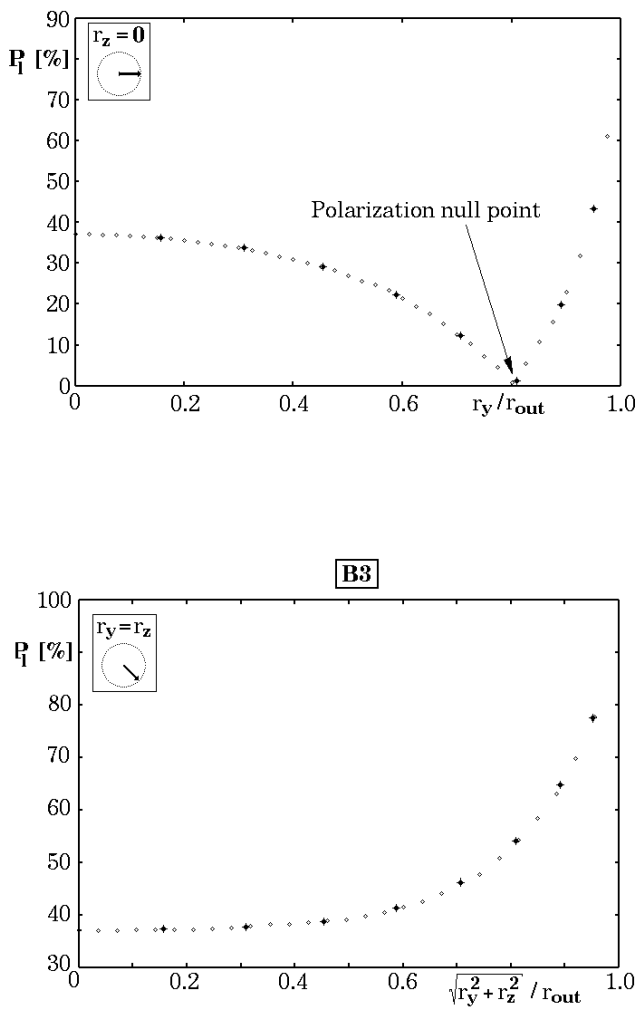

+ Matsumura \& Seki (1996) Monte-Carlo Simulation

Fig. 5. The results of test calculations for the comparison with the data of Matsumura \& Seki (1996). a) Polarization map of the configuration shown in Fig. 4. Only single scattering is considered. b1)-b3) The degree of linear polarization as a function of the projected distance from the centre along three different directions. The projected distance $r / r_{\text {out }}$ is connected with the scattering angle as $r / r_{\text {out }}=\arcsin \Theta$. b1) $P_{1}$ in the midplane of polarization map; b2) $P_{1}$ perpendicular to the midplane; b3) $P_{1}$ along a line being inclined by $45^{\circ}$ to the midplane.

Figs. 5a and 5b1). They have been observed in a number of objects (see discussion in Sect. 4.2.3). Sometimes, these null points are interpreted as the indicators for the "outer edge" of a circumstellar disk (transition from the optically thick disk to the optically thin shell; see Fischer et al. 1994). Although this explanation is very plausible, the simulations presented here show that uniformly aligned grains (possibly by an external magnetic field) may produce a similar polarization pattern. A detailed discussion of the polarization null points as a function of the model parameters is given in Sect. 4 .

\subsection{Multiple scattering}

To test the RT with multiple scattering, we consider model configurations for which the dust density distribution, the dust grain orientation, and the irradiation characteristic are centro-symmetric with respect to the observer's position. The resulting polarization pattern is therefore also centro-symmetric: the polarization vectors are oriented perpendicular or parallel to the radius vector of the configuration, in dependence of the refractive index and the grain size parameter $x_{\mathrm{V}}$. Thus, the integral (net) linear and circular polarization have to be zero. Because this property of centro-symmetric configurations does not depend on its optical thickness, it can be used as a test of the single and multiple scattering case.

We considered the following dust configurations containing either oblate or prolate grains:

1. Spherical dust shell with a point-like radiation source in the centre and particle rotation axes either parallel or perpendicular to the radius vector;

2. Cylindrical dust shell with a point-like radiation source on its rotation axis and particle rotation axes either parallel or perpendicular to the radius vector which is oriented perpendicular to the rotation axis of the cylinder.

While for the first configuration the net polarization is equal to zero for every position of the observer, for the second configuration this is the case only if the observer's line of sight is oriented perpendicular to one of the two 
cylinder footprints. In all cases we arrived at the expected results.

\section{Results and discussion}

In Sect. 4.1, light scattering in a geometrically thin hemisphere and sphere and a geometrically thick spherical shell is described. This preliminary modelling provides a "smooth" transition from the test models discussed in Sect. 3 to more realistic dust configurations. The basic modelling is presented in Sect. 4.2, where a geometrically thick shell is considered and the resulting polarization as a function of the main model parameters is investigated.

If one considers the RT in a medium consisting of spherical dust grains, scattering is the only mechanism to produce or to modify the polarization of the incident radiation. In the case of aligned spheroidal grains, the processes of photon absorption and re-emission may also fabricate or change polarization. Because of the obvious complexity of the problem, we separated the consideration of these three mechanisms of the polarization formation. The aim of the simulations discussed below was to outline the basic features of polarization arising from the light scattering by spheroidal grains. The additional influence of absorption and re-emission will be discussed in future publications.

For this reason, we apply the following approximations:

1. The grain albedo is assumed to be that of spherical grains with the equivolume radius $r_{\mathrm{V}}$ and is therefore a scalar. Then, the absorption process decreases the intensity of the incident radiation but does not modify the state of polarization;

2. We do not consider thermal re-emission of radiation.

If not stated differently, for the simulation presented in this section the dust grain and model parameters as described in Sect. 3 have been used. The observer's position is inclined by $i=90^{\circ}$ to the $z$-axis $\left(\tau_{z}(0.628 \mu \mathrm{m})=0.2\right)$ of the global coordinate system of the model space (see Fig. 4).

\subsection{Simple models}

Let us consider again the geometrically thin hemispherical model. While the polarization pattern in Fig. 5 is shown only for a position perpendicular to the $z$-axis $\left(i=90^{\circ}\right)$, Fig. 6 demonstrates how the behaviour of the degree of linear polarization in the midplane of the polarization map appears for different inclinations of the $z$-axis to the line of sight. It turns out that the local polarization minimum (the "polarization null point" or the reversal of polarization in the case $i=90^{\circ}$ ) are present at large inclination angles $i$ only. It is important to note that the polarization pattern (and therefore also the net polarization degree) for aligned non-spherical particles depends on the viewing angle even in the case of a one-dimensional density distribution. In contrast to this, no dependence of the

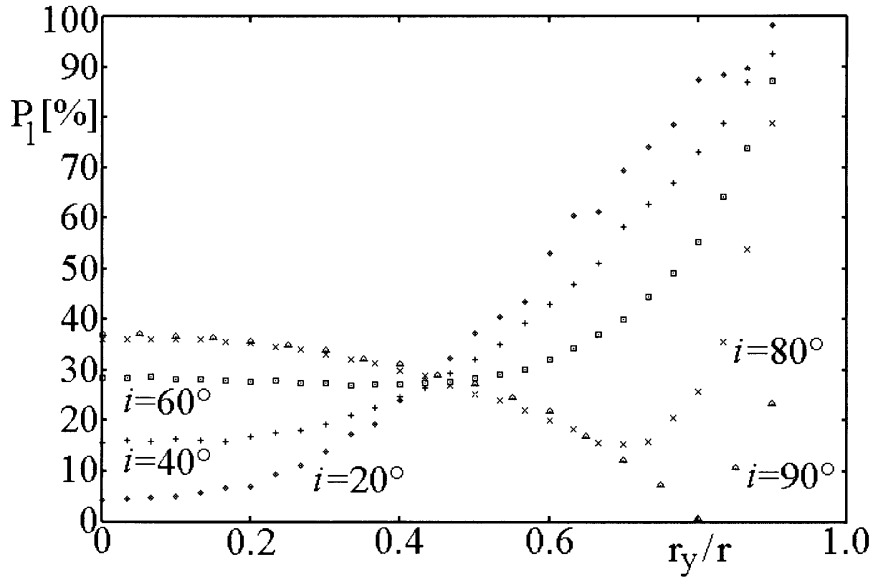

Fig. 6. The degree of linear polarization in dependence of the projected distance for tilted dust configurations.

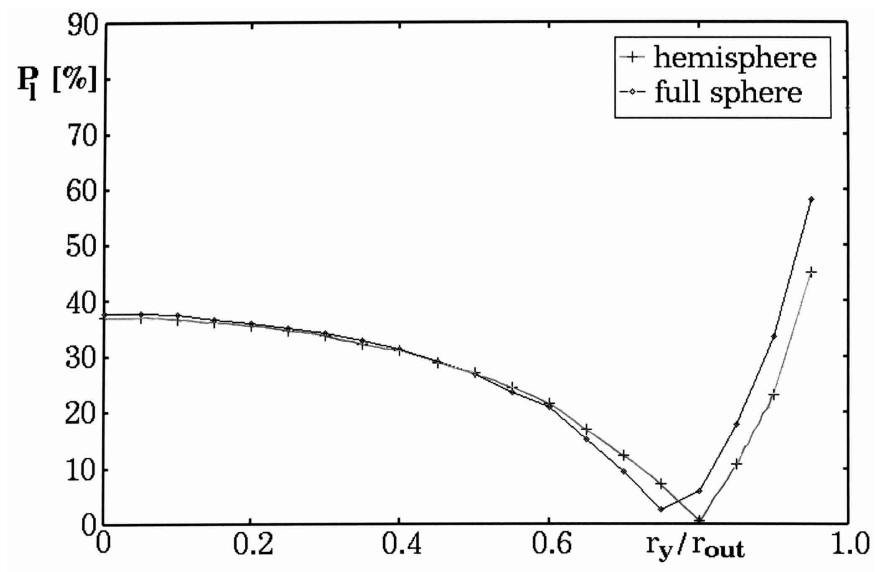

Fig. 7. The degree of linear polarization in dependence of the projected distance. Influence of the backward scattering is illustrated.

polarization on the inclination angle exists for spherical particles.

As a first step away from the very artificial configuration described above, we consider the RT in a thin spherical shell instead of the hemisphere shown in Fig. 4. Then, backward scattering in the hemisphere opposite to the observer's position (where $\Theta=90^{\circ}+\alpha$ ) has to be taken into account. The results are shown in Fig. 7. The addition of the backscattered radiation shifts the position of the minimum of the linear polarization to a smaller projected radius.

In Fig. 8, the circular polarization for this model of a geometrically and optically thin dust shell is shown. The circular polarization is equal to zero in the midplane and along the line through the centre perpendicular to the midplane. The net circular polarization from the configuration is also equal to zero. A counterpart of the linear polarization null points in the midplane could not be found. The maximum circular polarization is observed at the ends of lines inclined to the $z$-axis by $\pm 45^{\circ}$. These points correspond to the light scattering by particles with values of $\alpha=45^{\circ}, \Theta=90^{\circ}$ and $\Phi=90^{\circ}\left(270^{\circ}\right)$. 
[A]

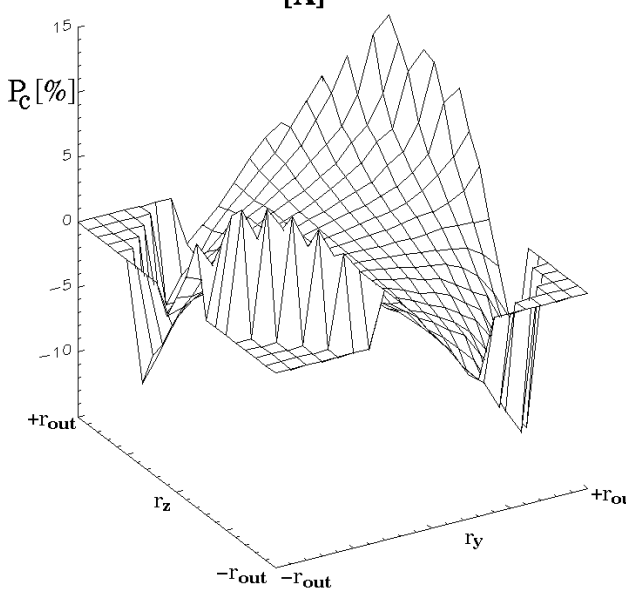

[B]

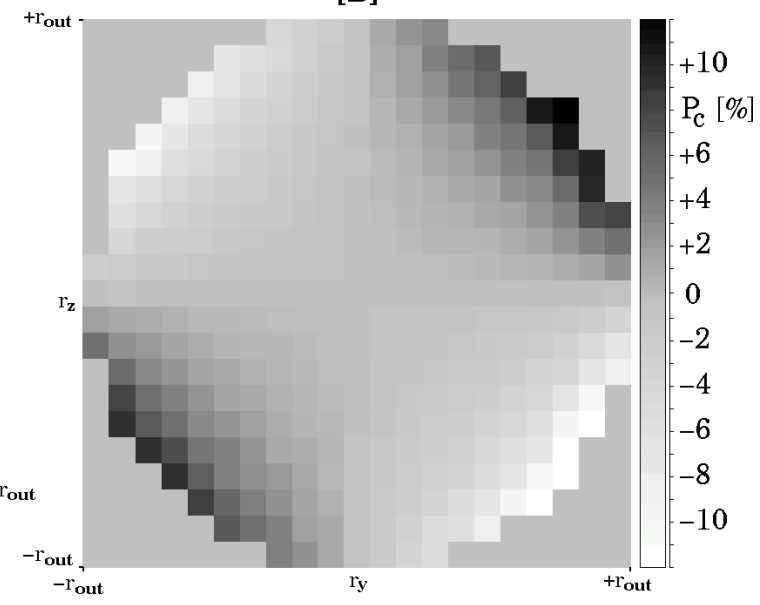

Fig. 8. a) The circular polarization of the scattered radiation arising from the configuration shown in Fig. 4. Deviations from the mirror symmetry result from the statistical noise of the Monte Carlo simulation. b) The same as grey plot to emphasize the symmetry of the spatial dependency of the circular polarization degree.

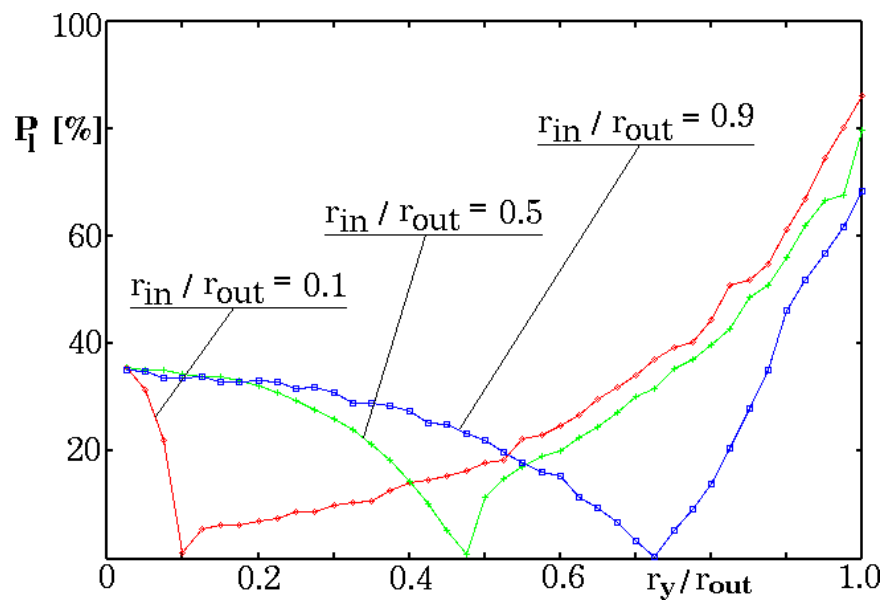

Fig. 9. The degree of linear polarization in dependence of the projected distance. The effect of the transition from a geometrically thin to a geometrically thick spherical shell is illustrated.

It turned out that the pattern of the linear polarization strongly depends on the inner radius of the spherical shell. This is demonstrated in Fig. 9, where the degree of linear polarization in the midplane is shown for three ratios of the inner to the outer radius of the shell. Decreasing this ratio (and therefore decreasing the relative size of the inner cavity around the illuminating star), the characteristic polarization null points shift towards the star. It has to be noted, that generally the polarization null point is not located at the position of the projected inner radius. The location, relative depth and width of the area where the polarization changes the sign depends on the geometrical thickness of the shell and grain parameters (see Sect. 4.2.2). The power-law density profile used (see Eq. (19)) leads to a preferred photon scattering near a narrow region at the inner boundary of the shell. The shift of the polarization null point is therefore mainly caused by the change of the inner radius $r_{\text {in }}$.

\subsection{Envelope models}

Now we will consider geometrically thick spherical shells. To represent such models, the ratio of the inner radius of the shell to the outer radius is fixed at $r_{\text {in }} / r_{\text {out }}=0.5$.

\subsubsection{Transition from optically thin to optically thick shell}

In the case of a small optical thickness, the main contribution to the resulting polarization is from first or second scattering events (Voshchinnikov \& Karjukin 1994; Voshchinnikov et al. 1995). As one can see from Fig. 10, the polarization $P_{1}\left(r_{y} / r_{\text {out }}\right)$ in the midplane decreases with increasing optical thickness. The same behaviour was found for the polarization perpendicular to the midplane $P_{1}\left(r_{z} / r_{\text {out }}\right)$. In general, the position of the local polarization minimum is not strongly influenced by the optical thickness.

Integrating the images for the Stokes parameters $I, Q, U$, we estimate the total linear polarization from our spherical dust configuration as one observes from the position perpendicular to the $z$-axis. Because of the symmetry, it is the same along the $x$ and $y$-axes

$P_{1,90^{\circ}}=\frac{F_{\mathrm{sca}, 90^{\circ}}^{\mathrm{p}}}{F_{\star} \exp \left[-\tau_{90^{\circ}}^{\mathrm{ext}}\right]+F_{\mathrm{sca}, 90^{\circ}}}$,

where $F_{\text {sca, } 90^{\circ}}$ and $F_{\text {sca, } 90^{\circ}}^{\mathrm{p}}$ are the scattered and polarized scattered radiation from the configuration. For the models presented in Fig. 10, the resulting polarization is $P_{1}=4 \%, 9 \%$, and $8 \%$, if $\tau_{z}=0.2,1$ and 2 , correspondingly. Excluding the stellar radiation (first term in the denominator in Eq. (20)), we can find the maximum degree of the observed linear polarization

$P_{1,90^{\circ}, \max }=\frac{F_{\mathrm{sca}, 90^{\circ}}^{\mathrm{p}}}{F_{\mathrm{sca}, 90^{\circ}}}$. 


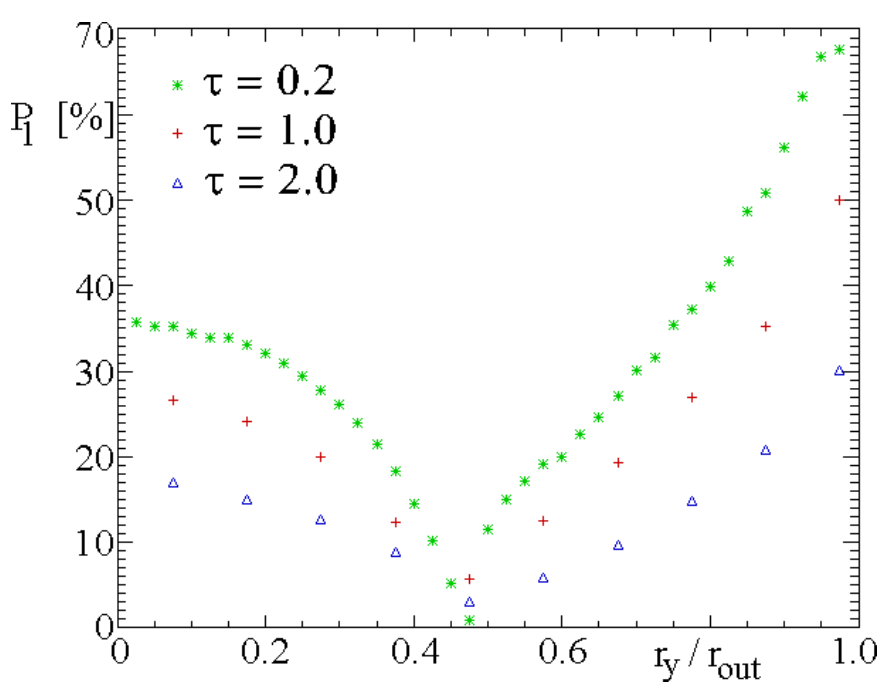

Fig. 10. The degree of linear polarization in dependence of the projected distance. The effect of the transition from an optically thin to an optically thick spherical shell is illustrated. Note that at this figure the resolution along the $y$-axis is much smaller than in comparison with other figures.

It is equal to $P_{1,90^{\circ}, \max }=25 \pm 1 \%, 15 \pm 1 \%$, and $9.1 \pm 1 \%$, if $\tau_{z}=0.2,1$ and 2, correspondingly.

\subsubsection{Dependence on dust grain parameters}

Figure 11 shows the midplane polarization $P_{1}\left(r_{y} / r_{\text {out }}\right)$ for oblate and prolate grains of three different equivolume radii $\left(r_{\mathrm{V}}=0.05 \mu \mathrm{m}, 0.1 \mu \mathrm{m}, 0.2 \mu \mathrm{m}\right)$. The behaviour of polarization for particles with $r_{\mathrm{V}}=0.05 \mu \mathrm{m}$ and $0.1 \mu \mathrm{m}$ is rather similar, while the polarization produced by the largest particles $\left(r_{\mathrm{V}}=0.2 \mu \mathrm{m}\right)$ is the smallest and is almost independent of the projected radius. This feature reflects the fact of decreasing polarizing efficiency of large particles (Voshchinnikov et al. 2000). Because the manner of polarization for prolate grains in the direction perpendicular to the midplane is similar to that for oblate grains in the midplane and vice versa (see also Fig. 13), the resulting polarization for particles of both types is quite similar. The net polarization - excluding the direct, non-scattered stellar radiation - amounts to $P_{1,90^{\circ} \text {, max }}=$ $20 \%(16 \%), 25 \%(20 \%)$ and $11 \%(10 \%)$ for oblate (prolate) grains with equivolume radii $r_{\mathrm{V}}=0.05 \mu \mathrm{m}, 0.1 \mu \mathrm{m}$ and $0.2 \mu \mathrm{m}$, respectively.

The distribution of the linear polarization $P_{1}\left(r_{y} / r_{\text {out }}\right)$ in the midplane of the spherical shell is plotted in Fig. 12 for particles with four different aspect ratios $a / b(a / b=$ $1.1,2,4,10)$. The polarization minima in the midplane $\left(r_{z}=0\right)$ appear for oblate grains only. Their position shifts to outer projected radii $r_{y} / r_{\text {out }}$ with increasing aspect ratio. If $a / b \rightarrow 1$, the location of the polarization null point leads to $r_{y} / r_{\text {out }} \rightarrow 0$. This is consistent with the behaviour of the degree of linear polarization for spherical grains where the null polarization can be found at the centre for the scattering angle $\Theta=0^{\circ}$. The resulting polarization for oblate and prolate grains of different shape
Table 1. The net linear polarization from the spherical shell containing perfectly aligned spheroidal grains $(m=1.7+0.03 i$, $\left.r_{\mathrm{V}}=0.1 \mu \mathrm{m}\right)$ as a function of the grains' axes ratio $a / b$. The direct stellar radiation is excluded.

\begin{tabular}{ccc}
\hline$a / b$ & Oblate particles & Prolate particles \\
\hline 1.1 & $3.6 \%$ & $3.0 \%$ \\
2 & $25 \%$ & $20 \%$ \\
4 & $43 \%$ & $31 \%$ \\
10 & $57 \%$ & $41 \%$ \\
\hline
\end{tabular}

is given in Table 1. Note that the polarization produced by oblate particles is larger in all cases.

The intensity maps of our configuration $\left(i=90^{\circ}\right)$ show an additional effect. In Fig. 13, the images with overlaid polarization pattern, corresponding to the intensity and linear polarization of scattered light, are shown for oblate and prolate grains with the aspect ratios $a / b=1.1$ and 2 . In the case of nearly spherical grains $(a / b=1.1)$, the ring-like structure is clearly seen for oblate as well as for prolate grains. Otherwise, in the case of $a / b=2$ the intensity maxima appear at the points of intersection with the $z(y)$-axis for oblate (prolate) grains. In both cases, the corresponding axis is parallel to the minor $(b)$ axis of grains. Increasing the aspect ratio $a / b$, this effect is strengthened. Note that the net polarization from the spherical configuration arises in the inner parts of the shell because the polarization pattern from the outer regions is centrosymmetric and the polarization would be cancelled. The direction of the resulting net polarization is parallel to the major $(a)$ axis of grains that is similar to the case of dichroic polarization.

\subsubsection{Null points on polarization maps as indicators of scattering by non-spherical grains}

The model discussed in this paper is still too simple in order to make a detailed comparison with observations. However, we will discuss qualitatively the behaviour of null points produced by scattering on non-spherical grains.

Polarization null points were observed in a variety of objects ranging from reflection nebula and circumstellar structures to external galaxies (e.g., Scarrott et al. 1989; Scarrott et al. 1990; Gledhill \& Scarrott 1989; Asselin et al. 1996; Chrysostomou et al. 1996; Hajjar \& Bastien 1996; Kastner \& Weintraub 1996; Wood \& Jones 1997; Gledhill et al. 2001). They appear approximately symmetrically on either side of the central illuminating source ${ }^{3}$. In all cases the origin of null points was attributed to the combined action of two polarization mechanisms, where the first (initial or internal) mechanism produced linearly polarized radiation whereas the

\footnotetext{
${ }^{3}$ It should be mentioned that the measurable polarization especially near the position of polarization null points - sensitively depends on the spatial resolution of the polarization maps. Therefore, it is more likely to find the polarization minima but not real null points.
} 

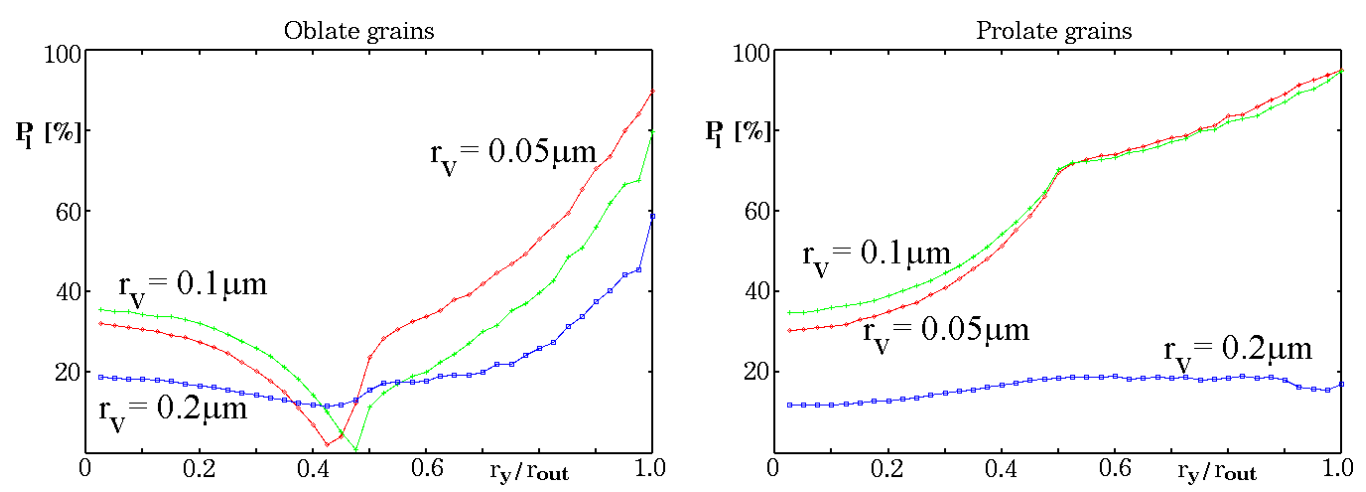

Fig. 11. The degree of linear polarization in dependence of the projected distance. The effect of the variations of the equivolume radius $r_{\mathrm{V}}$ for oblate and prolate grains is illustrated $\left(r_{\mathrm{V}}=0.05 \mu \mathrm{m}, 0.1 \mu \mathrm{m}, 0.2 \mu \mathrm{m}\right)$.
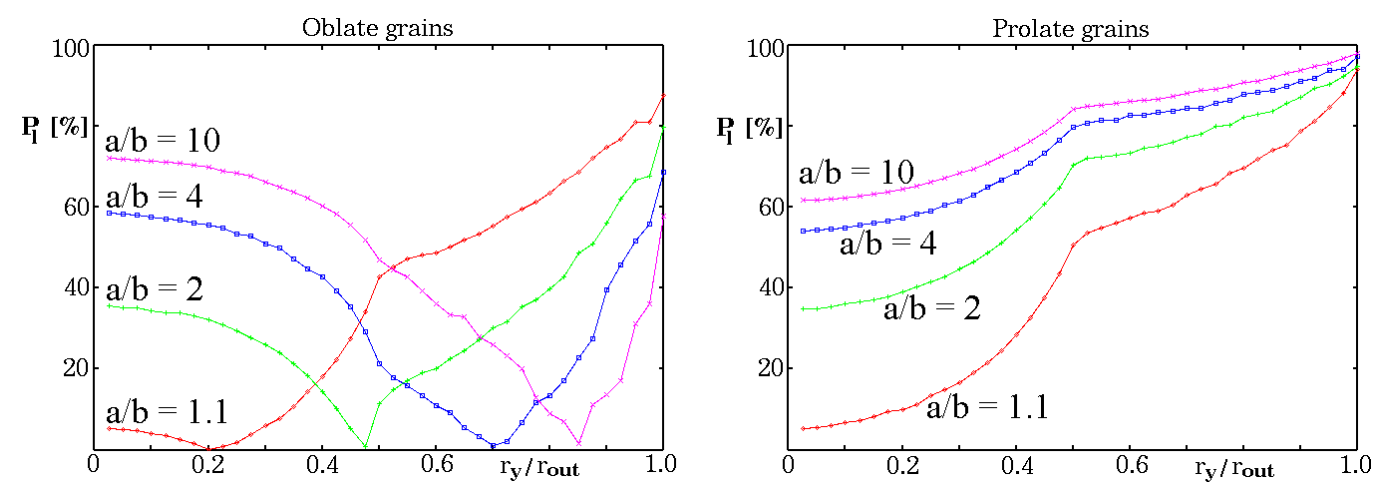

Fig. 12. The degree of linear polarization for the case of oblate and prolate grains in dependence of the projected distance. The effect of the variations of the grain shape (the aspect ratio) is illustrated $(a / b=1.1,2,4,10)$.

second (external) mechanism led to the compensation of this polarization. Three possible models were qualitatively discussed by Scarrott et al. (1989):

(i) polarization due to scattering by spherical grains and its cancellation via dichroic extinction in an external medium consisting of aligned non-spherical particles;

(ii) polarization due to scattering by spherical grains in an optically thick internal medium and its cancellation after the next scattering by spherical grains in an external optically thin medium;

(iii) polarization of light from the central source due to an unspecified mechanism and its compensation due to the second scattering by spherical grains in an external medium.

These models could be called "hybrid models" because they require two steps to produce the observed polarization null points. The model (i) is rather naturally applied to the modelling of polarization maps of galaxies (Wood \& Jones 1997). The multiple scattering model (ii) was used by Bastien \& Ménard (1988) and Fischer et al. (1994) for interpretation of polarization maps of young stellar objects. In this case, the centrosymmetric polarization pattern is obtained in outer optically thin parts of the shell, whereas the aligned polarization vectors appear in the dense inner parts of this shell. The polarized source model (iii) was discussed by Notni (1985) and Gledhill (1991). Using this model, it is possible to explain the polarization pattern observed in several bipolar and cometary nebu- lae (e.g., Gledhill 1991), but as it was shown by Clark et al. (2000) the models with spherical grains fail to produce even the $5 \%$ circular polarization found in the reflection nebulosity around the Herbig Ae/Be star R CrA. In this case, the authors suggested scattering by aligned non-spherical grains as the operating mechanism.

In contrast to the hybrid models, different polarimetric effects can arise as a result of light scattering by aligned non-spherical particles. In our simple model, the location of polarization null points depends on the particle shape, size and the inner to outer radius ratio (see Figs. 9, 11 and 12). The wavelength dependence of the location of null points is not very strong and their position only slightly approaches the central (stellar) position when going from the $V$ to $K$ band as it follows from Fig. $14^{4}$. A similar conclusion can be reached from the observed polarization maps of the variable Hubble's nebula NGC 2261 (Scarrott et al. 1989) and the infrared reflection nebula GSS 30 (Chrysostomou et al. 1996). The results of Asselin et al. (1996) for V376 Cas show that the position of the null points is also shifted as a function of the binned box size used in averaging the polarization. However, it should

\footnotetext{
${ }^{4}$ In the case of polarization null points which have been observed at the location of the outer boundary of circumstellar disks around young stellar objects (transition from the optically thick disk to the optically thin shell), the same behaviour is expected since this boundary is slightly shifted inward with increasing wavelength.
} 


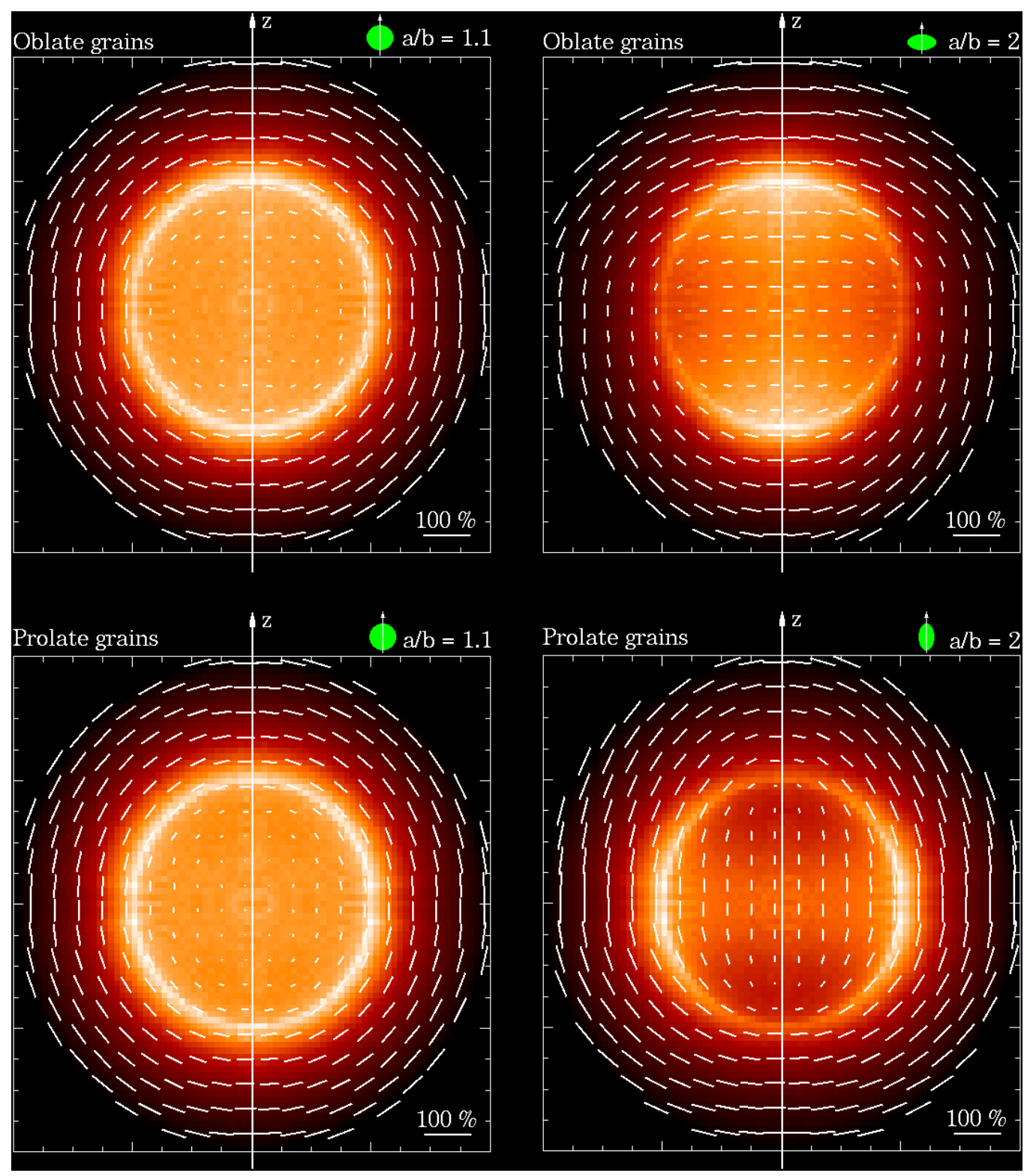

Fig. 13. Intensity maps with overlaid polarization pattern of a spherical shell containing perfectly aligned dust grains (for the description of the model configuration see Sect. 4.2.2). Due to chosen shell and grain parameters, the brightest knots appear at the inner boundary of the shell.

be noted that near the null points the observed signal is usually very small and the errors are large.

Finally, we can indicate that the scattering by almost spherical grains is marked by a ring-like structure in the intensity maps whereas the null points are located inside this structure rather close to the stellar position (Fig. 13, left side). In the case of aligned non-spherical particles (Fig. 13, right side), the null points at the inner boundary of the spherical shell are accompanied by symmetric arclike structures in the intensity maps. There is some similarity of such maps with those of small reflection nebulae published by Gledhill et al. (2001): the "spherical case" is seen in the source IRAS $19114+0002$ while the "nonspherical case" occurs in the maps of IRAS $22223+4327$, IRAS 19500-1709 and IRAS 17106-3046.
We should emphasize that in the presented model the origin of null points is caused by light scattering by nonspherical particles, but is not related to the cancellation of polarization as in hybrid models.

\section{Conclusions}

The paper contains the first solution to the RT problem including multiple light scattering in dust configurations with aligned non-spherical dust grains. Our main aim was to discuss the effects of light scattering by spheroidal dust grains in a simple (spherical) dust configuration, containing perfectly aligned particles.

The most remarkable features of the simulated linear polarization maps are the polarization null points where 


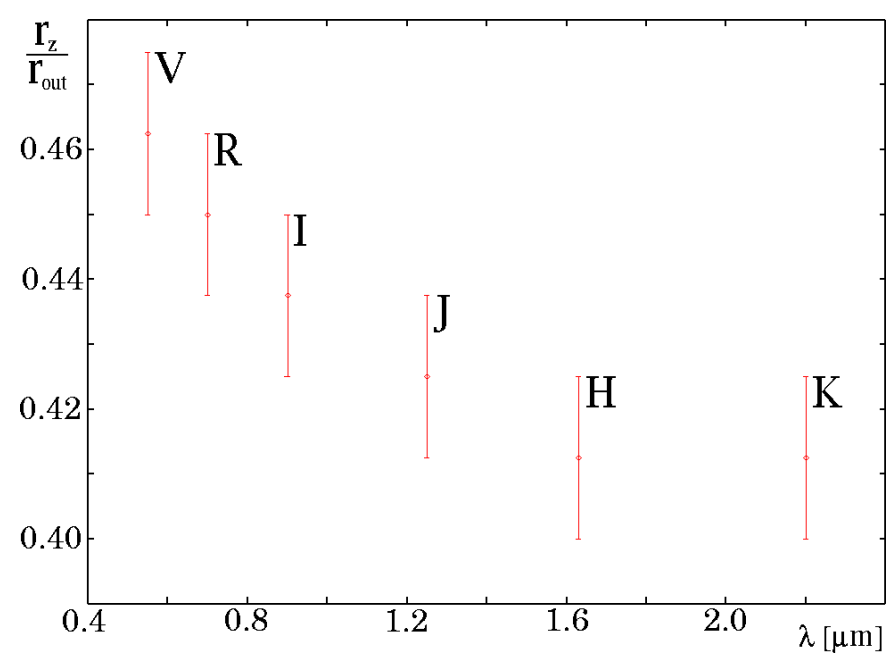

Fig. 14. Projected positions of null points on polarization maps in dependence of wavelength and their statistical errors for the case prolate grains with aspect ratio $a / b=2$ (the similar model is shown in Fig. 13, right lower panel). The calculations were performed for effective wavelengths of Johnson photometric system.

the reversal of polarization occurs. They appear when the grain alignment axis is perpendicular to the line of sight. Symmetrically to the polarization null points, we found maxima in the intensity maps.

In contrast to spherical grains, even single scattering by spheroidal grains may cause circular polarization. The maps of circular polarization have a sector-like structure with polarization maxima at the ends of a line inclined to the grain alignment axis by $\pm 45^{\circ}$.

Based on our theoretical investigations of light scattering, dichroic absorption, and (re)emission by spheroidal grains presented in Sect. 2, the next steps of the numerical radiative transfer simulations will include the consideration of light scattering by partly aligned rotating particles and the calculation of the polarized re-emission of grains in different dust configurations. This will immediately lead to an improved interpretation and therefore better understanding of mid-infrared and submillimeter polarization where re-emission and dichroic absorption by partly aligned non-spherical dust grains are the main polarization mechanisms. Thus, it provides a basis for investigations of the structure of the magnetic fields in these objects which are in most cases assumed to cause grain alignment.

Acknowledgements. We appreciate the discussions of light scattering by non-spherical particles with Victor Farafonov and Bernhard Michel and wish to thank M. Matsumura for providing his results for comparison. We are grateful to the referee for constructive comments. This research was supported by the DFG grant Ste 605/10 within the program "Physics of star formation" and by grants of the Volkswagen Foundation and INTAS (Open Call 99/652).

\section{References}

Asselin, L., Ménard, F., Bastien, P., et al. 1996, ApJ, 472, 349

Bastien, P., \& Ménard, F. 1988, ApJ, 326, 334

Bohren, C. F., \& Huffman, D. R. 1983, Absorption and scattering of light by small particles (John Wiley \& Sons, New York)

Chrysostomou, A., Clark, S. G., Hough, J. H., et al. 1996, MNRAS, 278, 449

Chrysostomou, A., Gledhill, T., Ménard, F., et al. 2000, MNRAS, 312, 103

Clark, S., McCall, A., Chrysostomou, A., et al. 2000, MNRAS, 319, 337

Daniel, J.-Y. 1980, A\&A, 87, 204

Dollfus, A., \& Suchail, J.-L. 1987, A\&A, 187, 669

Elvius, A., \& Hall, J. S. 1967, Lowell Obs. Bull., 7, 17

Farafonov, V. G., Il'in, V. B., \& Prokop'eva, M. S. 2001, Opt. spektrosc., submitted

Fischer, O., Henning, Th., \& Yorke, H. W. 1994, A\&A, 284, 187

Gledhill, T. 1991, MNRAS, 252, 138

Gledhill, T., \& McCall, A. 2000, MNRAS, 314, 123

Gledhill, T., \& Scarrott, S. M. 1989, MNRAS, 236, 139

Gledhill, T. M., Chrysostomou, A., Hough, J. H., \& Yates, J. A. 2001, MNRAS, 322, 321

Hajjar, R., \& Bastien, P. 1996, in ASP Conf. Ser., 97, Polarimetry of the Interstellar Medium, ed. W. G. Roberge, \& D. C. B. Whittet, 355

Hovenier, J. W., \& van der Mee, V. M. 2000, in Light Scattering by Nonspherical Particles, ed. M. I. Mishchenko, et al. (Academic Press, San Francisco), 61

Johnson, J. J., \& Jones, T. J. 1991, AJ, 101, 1735

Kastner, J. H., \& Weintraub, D. A. 1996, in ASP Conf. Ser., 97, Polarimetry of the Interstellar Medium, ed. W. G. Roberge, \& D. C. B. Whittet, 212

Kim, S. H., \& Martin, P. G. 1995, ApJ, 444, 293

Martin, P. G. 1974, ApJ, 187, 461

Matsumura, M., \& Seki, M. 1996, in ASP Conf. Ser. 97, Polarimetry of the Interstellar Medium, ed. W. G. Roberge, \& D. C. B. Whittet, 63

Matsumura, M. 1999, private communication

Ménard, F. 1988, Ph.D. Thesis, Université de Montréal

Notni, P. 1985, AN, 306, 265

Onaka, T. 2000, ApJ, 533, 298

Scarrott, S., Draper, P. W., \& Warren Smith, R. F. 1989, MNRAS, 237, 621

Scarrott, S., Rolph, C. D., \& Semple, D. P. 1990, in IAU Symp. 140, Galactic and Intergalactic Magnetic Fields, ed. R. Beck, \& P. P. Kronberg (Dordrecht: Kluwer), 245

Voshchinnikov, N. V. 1990, SvA, 34, 535

Voshchinnikov, N. V. 1998, IAU Symp., 191, 3

Voshchinnikov, N. V., \& Farafonov, V. G. 1993, Ap\&SS, 204, 19

Voshchinnikov, N. V., \& Karjukin, V. V. 1994, A\&A, 288, 883

Voshchinnikov, N. V., Grinin, V. P., \& Karjukin, V. V. 1995, A\&A, 294, 554

Voshchinnikov, N. V., Il'in, V. B., Henning, Th., et al. 2000, JQSRT, 65, 877

Wolf, S., Fischer, O., \& Pfau, W. 1998, A\&A, 340, 103

Wolf, S., \& Henning, Th. 2000, Computer Physics Comm., 132, 166

Wolf, S., Henning, Th., \& Stecklum, B. 1999, A\&A, 349, 839

Wood, K., \& Jones, T. J. 1997, AJ, 114, 1405 